\title{
Bardet-Biedl syndrome
}

\begin{abstract}
Bardet-Biedl syndrome (BBS) is a rare autosomal recessive ciliopathy characterised by retinal dystrophy, obesity, post-axial polydactyly, renal dysfunction, learning difficulties and hypogonadism. Many associated minor features can be helpful in making a diagnosis and are important in the clinical management of BBS. The diagnosis is based on clinical findings and can be confirmed by sequencing of known disease-causing genes in $80 \%$ of patients. BBS genes encode proteins that localise to the cilia and basal body and are involved in cilia biogenesis and function. Mutations lead to defective cilia accounting in part for the pleiotropic effects observed in BBS. We provide an overview of BBS including the clinical findings, current understanding of cilia biology, and a practical approach to diagnosis, genetic counselling and up-to-date management.
\end{abstract}

\section{In Brief}

- Autosomal recessive ciliopathy.

- The estimated incidence is 1:160000 in northern European populations and 1:13500 in some Arab populations.

- Characterised by retinal dystrophy, renal dysfunction, post-axial polydactyly, obesity, cognitive deficit and hypogenitalism.
- Diagnosis is based on clinical features.

- Molecular genetic testing is available and currently 16 genes are known to be associated with Bardet-Biedl syndrome (BBS), accounting for approximately $80 \%$ of clinically diagnosed BBS.

- Surveillance includes regular ophthalmological evaluation, montoring of renal, liver, glucose, lipid and endocrine profile and regular weight and blood pressure measurements.

\section{INTRODUCTION}

In 1866, Laurence and Moon described a family of four siblings with retinal dystrophy, obesity, spastic paraparesis and cognitive deficit. ${ }^{1}$ Bardet $^{2}$ and Biedl $^{3}$ later reported separately on further similarly affected individuals who in addition had post-axial polydactyly and the condition was coined Laurence-Moon-Bardet-Biedl syndrome. The syndrome is often divided into two entities: Laurence-Moon syndrome and Bardet-Biedl Syndrome (BBS), but there is considerable phenotypic overlap, suggesting that they may be allelic. ${ }^{4}$ BBS is now the standard term in common usage.

BBS is a pleiotropic genetic disorder with significant interfamilial and intrafamilial variation. ${ }^{5,6}$ Inheritance is traditionally considered autosomal recessive, although notable exceptions exist, whereby BBS may be an oligogenic disorder. ${ }^{7}$ The prevalence of BBS varies markedly between populations; from 1:160 000 in northern European populations $^{5}$ to $1: 13500$ and 1:175000, respectively, in isolated communities in Kuwait and Newfoundland, where a higher level of consanguinity prevails. ${ }^{8,9}$ Studies on the families in Newfoundland affected with BBS revealed a minimum of six BBS loci and eight different BBS mutations and therefore the high prevalence cannot be attributed to a single founder. ${ }^{10}$

Elizabeth Forsythe ${ }^{1}$ and Philip L Beales ${ }^{*, 1}$

${ }^{1}$ Molecular Medicine Unit, Institute of Child Health, University College London, London, UK

European Journal of Human Genetics (2013) 21, 8-13; doi:10.1038/ejhg.2012.115; published online 20 June 2012

Keywords: Bardet-Biedl syndrome; ciliopathies; autosomal recessive inheritance; BBS genes

*Correspondence: Professor PL Beales, Molecular Medicine Unit, Institute of Child Health, University College London, 30 Guilford Street, London WC1N 1EH, UK. Tel: +44 207905 2159; Fax: +44 207404 6191; E-mail: p.beales@ucl.ac.uk

Received 20 September 2011; revised 29 March 2012; accepted 4 April 2012; published online 20 June 2012

\section{CLINICAL OVERVIEW}

The BBS phenotype evolves slowly throughout the first decade of life, although there is a considerable variability. As a result, most patients are diagnosed in late childhood or early adulthood. Post-axial polydactyly is common and may be the only obvious dysmorphic feature at birth. This may affect all four limbs or only upper or lower limbs and may occur in conjunction with brachydactyly and/or syndactlyly.

The most common diagnostic handle prompting investigation for BBS is the development of rod-cone dystrophy. Primary loss of rod photoreceptors is followed by later demise of cone photoreceptors. ${ }^{11}$ This presents as an atypical retinitis pigmentosa with early macular involvement. ${ }^{12}$ The clinical manifestation is gradual onset of night blindness, followed by photophobia and loss of central and colour vision. ${ }^{11}$ Many variants of the ophthalmological phenotype in BBS have been described and some patients develop the converse sequence of pathological events with early loss of cone photoreceptors followed by rod photoreceptors. ${ }^{4}$ Electroretinography is the investigation of choice and may show early changes within the first two years of life, although significant changes are rarely visible before the age of five. ${ }^{12}$ Symptoms usually develop in the first decade of life and most patients are legally blind by the second or third decade, ${ }^{13}$ although moderate forms of the disease do exist. Other eye abnormalities such as cataracts and refractive errors are also prevalent in BBS.

Obesity is another major clinical finding and the incidence is reported to be $72-86 \%$ in the BBS population. ${ }^{4,6,14-17}$ Birth weight is usually within the normal range, although there is an evidence of a skewed distribution towards the upper centiles. ${ }^{18}$ One-third of those with a normal birth weight develop obesity by the age of one. ${ }^{18}$ Although adult obesity tends to be truncal, it appears to be widespread and diffuse in childhood.

The development of type 2 diabetes is prevalent among patients. It may be related to the level of obesity and it is often found in association with other signs of metabolic syndrome.

Hypogonadism may manifest as delayed puberty or hypogenitalism in males and genital abnormalities in females. ${ }^{4,17,19}$ This may occur 
independently or in conjunction with biochemical hypogonadism. A wide variety of genital malformations have been observed in females, contributing to the low rates of fertility in BBS. Males are almost invariably infertile.

Developmental delay and cognitive deficit are common in BBS. Delay is often global but may be specific to certain areas of development. ${ }^{12}$ In a cohort of 109 patients, cognitive deficit was reported in $62 \%$ and half of these patients attended a special school. ${ }^{4}$ Children with BBS are often reported to have labile behaviour with outbursts of frustration. ${ }^{4,17,19}$ Many prefer to have a fixed routine and may have elements of obsessive compulsive behaviour and lack of social dominance. ${ }^{4}$ Others have a more severe behavioural phenotype and develop autistic spectrum disorder or psychosis. ${ }^{4}$

Renal abnormalities can be a major cause of morbidity and mortality in BBS. ${ }^{20}$ The renal phenotype is variable but classically manifests with cystic tubular disease and anatomical malformations. ${ }^{18}$ Urinary concentration defects are prevalent even in patients with near-normal renal function and no major cysts. ${ }^{21}$

Speech deficit has been reported in $60 \%$ of patients. ${ }^{22}$ This mainly consists of high-pitched nasal speech and children often do not develop intelligible speech before the age of four. It has been suggested that substitution of the first consonant of a word may be characteristic. ${ }^{4}$ Speech difficulties may be complicated by hearing loss, which is reported in $17-21 \%$ of patients. ${ }^{4,19}$ Most patients suffer conductive hearing loss secondary to chronic otitis media. ${ }^{4}$ Speech delay in children with BBS is generally responsive to speech therapy. 4

Involvement of other organ systems such as the heart and gastrointestinal system are also observed. The type of cardiac abnormalities observed in BBS is highly variable. An echocardiography study of 22 individuals from three highly consanguineous Bedouin families revealed a frequency of heart defects of $50 \%{ }^{23}$ Beales et al ${ }^{4}$ found a frequency of only 7\% in a study based on 109 patients. Cardiac abnormalities include valvular stenoses, patent ductus arteriosis and cardiomyopathies. ${ }^{4}$ Hepatic involvement ranges from fibrosis to cystic dilatation of the bile duct, intrahepatic and extrahepatic tracts. ${ }^{12}$ Hirschsprungs disease has been documented in BBS but the incidence of this association is unknown. ${ }^{24}$

Dental crowding and a high-arched palate are common. Other abnormalities include hypodontia, malocclusion and enamel hypoplasia. ${ }^{5}$ Anosmia has been described following observations in a mouse model. In a study of 19 BBS patients, nine had anosmia/ hyposmia. ${ }^{25}$

Many affected individuals suffer from a degree of clumsiness and $40 \%$ of one cohort describe signs of ataxia and poor coordination. ${ }^{4}$ Dysdiadochokinesia and past pointing are common (79\%) as are difficulties with tandem walking and the Fogg test. ${ }^{4}$

There is a significant overlap in clinical features within the ciliopathy disease spectrum. Although the classical features associated with BBS are well documented, it has been suggested that the BBS phenotype may be even more confluent with other ciliopathies such as Alström and McKusick-Kauffman syndromes than previously reported. ${ }^{19}$ Alström syndrome is usually differentiated from BBS by the presence of hearing loss and absence of polydactyly and learning difficulties. Patients with McKusick-Kauffman syndrome have a high prevalence of urogenital anomalies but normally lack the obesity, rod-cone dystrophy, and learning difficulties characteristic of BBS. Diagnostic difficulties arise as these features are agedependent in $\mathrm{BBS}^{26}$

Carriers of BBS do not appear to be at increased risk of hypertension or diabetes, ${ }^{4,10}$ although conflicting evidence exists regarding the possible increased risk of obesity in carriers. $4,10,27,28$ An increase in renal cancers and malformations, ${ }^{29}$ as well as retinal dysfunction, ${ }^{30}$ has been reported in obligate carriers.

Of note, mutation analysis in non-BBS lean and obese Caucasian patients revealed an association between single-nucleotide polymorphisms in BBS2, BBS4 and BBS6 and common obesity. ${ }^{31}$

\section{DIAGNOSTIC APPROACH}

Unless the diagnosis is suspected based on antenatal imaging revealing polydactyly and structural renal abnormalities, BBS is usually not diagnosed before the patient starts to develop the visual problems characteristic of rod-cone dystrophy. Although there are some distinctive dysmorphic features such as hypertelorism, midface hypoplasia and retrognathia, these are inconsistently present and can be subtle. Figure 1 demonstrates the variation of facial features seen in BBS. Modified diagnostic criteria by Beales et al ${ }^{4}$ suggest that either four primary features or three primary and two secondary features are required to make a clinical diagnosis. Table 1 summarises the major and minor clinical features and their incidence in BBS.

Molecular confirmation of the diagnosis can be obtained in nearly $80 \%$ via direct sequencing of the BBS genes. Billingsley et $a^{32}$ have suggested a practical approach to mutational screening where sequencing is prioritised in accordance with the frequency of pathogenic mutations between and within BBS genes in order to provide an efficient and cost-effective service.

\section{GENETIC BASIS OF THE DISEASE}

The last decade has seen a rapid expansion in research into this syndrome, resulting in the discovery of 16 BBS genes accounting for approximately $80 \%$ of clinically diagnosed BBS (Table 2). The majority of pathogenic mutations are found in BBS1 and BBS10, accounting for $23.2 \%$ and $20 \%$, respectively, ${ }^{5}$ although some regional variation in prevalence exists. ${ }^{15}$ Some genes appear to have greater ethnic specific frequency than others, although no mutated genes are found exclusively in a single ethnic population. In northern European individuals, BBS1 M390R and BBS10 C91LfsX5 are the most common alleles, whereas mutations in BBS4, BBS5 and TTC 8 are mainly seen in patients of Middle Eastern and North African descent. ${ }^{32}$ Although traditionally considered an autosomal recessive condition, there are several reported cases of a triallelic mode of inheritance where three mutations in BBS genes are required before the phenotype becomes apparent, or alternatively where a third disease locus acts as a disease modifier. ${ }^{7,33-36}$

As well as the phenotypic overlaps exhibited between ciliopathies, there is an emerging evidence suggesting that genes mutated in BBS and other ciliopathies exhibit some genetic overlap. ${ }^{37}$ For example, mutations in BBS2, BBS4 and BBS6 have been identified in patients with Meckel syndrome. ${ }^{38}$ Similarly, mutations in MKS1, which normally lead to Meckel syndrome, may be associated with a BBS phenotype and mutations in MKS3 have been identified in patients with BBS and Joubert syndrome. ${ }^{39}$ It appears that the ciliopathy phenotype reflects both the specific mutated loci and the total mutational load. ${ }^{39,40}$

\section{Genotype-phenotype correlation}

Genotype-phenotype correlations are poor, although one study suggests a milder phenotype associated with the common M390R mutation found in $B B S 1 .{ }^{15}$ Other studies have suggested that specific ocular phenotypes ${ }^{41,42}$ and more severe digital abnormalities ${ }^{42}$ may be linked to BBS2, BBS3 and BBS4 mutations. Large-scale studies have not supported these findings, and predictions about correlation are 

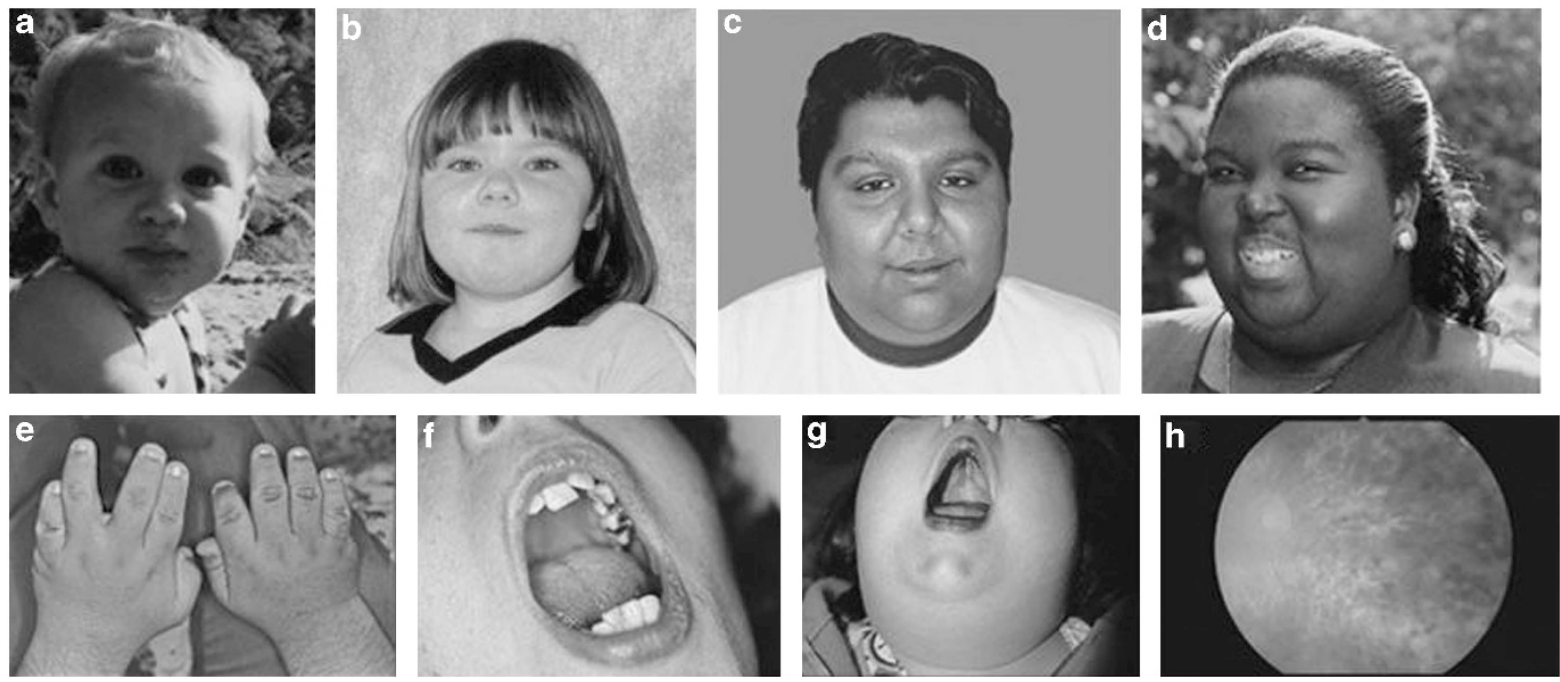

Figure 1 Images of patients demonstrating the dysmorphic features associated with BBS. (a-d) Demonstrate typical facial features. These are often subtle and are not always present. Features include deep-set eyes, hypertelorism, downward slanting palpebral fissures, a flat nasal bridge, small mouth, malar hypoplasia and retrognathia. (e) Brachydactyly and scars from excision of accessory digits. (f) Dental crowding. (g) High-arched palate. (h) Fundoscopy demonstrating rod-cone dystrophy.

Table 1 Diagnostic features and prevalence in BBS

\begin{tabular}{ll}
\hline & Frequency \\
\hline Primary features & \\
Rod-cone dystrophy & $93 \%$ \\
Polydactyly & $63-81 \%$ \\
& All four limbs: 21\% \\
& Upper limbs only: $9 \%$ \\
& Lower limbs only:21\% \\
Obesity & $72-92 \%$ \\
Genital anomalies & $59-98 \%$ \\
Renal anomalies & $53 \%$ \\
Learning difficulties & $61 \%$ \\
Secondary features & \\
Speech delay & \\
Developmental delay & $54-81 \%$ \\
Diabetes mellitus & $50-91 \%$ \\
Dental anomalies & $6-48 \%$ \\
Congenital heart disease & $51 \%$ \\
Brachydactyly/ syndactyly & $7 \%$ \\
Ataxia/ poor coordination & $46-100 \% / 8-95 \%$ \\
Anosmia/hyposmia & $40-86 \%$ \\
\hline Four primary featur or threp & $60 \%$ \\
\hline
\end{tabular}

Four primary features or three primary features and two secondary features are required for a clinical diagnosis of Bardet-Biedl syndrome. ${ }^{4,16,18}$

further compounded by the significant interfamilial and intrafamilial phenotypic variability. This supports the hypothesis that the BBS proteins interact in a common cellular process, thus making the genotypes clinically indistinguishable. ${ }^{37}$

Submission of BBS gene variants to a public databases such as Mutadatabase (www.mutadatabase.org) may aid in further delineation of the genotype-phenotype correlation.

\section{BIOLOGY OF THE DISEASE}

Cilia are highly conserved cellular structures projecting from the apical surface of most vertebral cells. They fall into two classes: motile and immotile (primary) cilia. ${ }^{12,14}$ Motile cilia are organised in a ' $9+2$ ' microtubule configuration in which nine microtubule pairs
Table 2 BBS genes

\begin{tabular}{|c|c|c|c|}
\hline Gene & Frequency & Locus & Function \\
\hline$B B S 1$ & $23 \%$ & $11 q 13$ & BBSome protein \\
\hline BBS2 & $8 \%$ & $16 q 21$ & BBSome protein \\
\hline$B B S 3 / A R L 6$ & $0.4 \%$ & $3 p 12-p 13$ & GTPase \\
\hline$B B S 4$ & $2 \%$ & 15q22.3-q23 & BBSome protein \\
\hline BBS5 & $0.4 \%$ & $2 q 31$ & BBSome protein \\
\hline BBS6/MKKS & $6 \%$ & $20 p 12$ & Part of chaperonin complex \\
\hline BBS7 & $2 \%$ & 4q27 & BBSome protein \\
\hline BBS8/TTC8 & $1 \%$ & $14 q 32.1$ & BBSome protein \\
\hline$B B S 9 / B 1$ & $6 \%$ & $7 p 14$ & BBSome protein \\
\hline$B B S 10$ & $20 \%$ & $12 q 21.2$ & Part of chaperonin complex \\
\hline BBS11/TRIM32 & $0.1 \%$ & $9 q 31-q 34.1$ & E3 ubiquitin ligase \\
\hline BBS12 & $5 \%$ & $4 q 27$ & Part of chaperonin complex \\
\hline BBS13/ MKS1 & $4.5 \%$ & $17 q 23$ & Centriole migration \\
\hline $\begin{array}{l}\text { BBS } 14 / C E P 290 / \\
\text { NPHP6 }\end{array}$ & $1 \%$ & $12 q 21.3$ & Basal body: RPGR interaction \\
\hline BBS15/ WDPCP & $1 \%$ & $2 \mathrm{p} 15$ & $\begin{array}{l}\text { Basal body: localisation of septins } \\
\text { and ciliogenesis }\end{array}$ \\
\hline BBS16/SDCCAG8 & $1 \%$ & $1 q 43$ & Basal body: interacts with OFD1 \\
\hline
\end{tabular}

surround a central doublet. These cilia generate flow or movement of fluid. Figure 2 outlines the structure of the motile and immotile cilium. Defective motile cilia can result in primary ciliary dyskinesias manifesting with bronchiectasis, infertility and left-right asymmetry. ${ }^{43}$ Immotile (primary) cilia are thought to function mainly as a sensory organelle regulating the signal transduction pathways and have a ' $9+0$ ' configuration, similar in structure to the motile cilia but without the central pair. ${ }^{12,14,44}$ Defects in immotile cilia are characterised clinically by retinitis pigmentosa, polydactyly, situs inversus, learning difficulties and cystic kidneys, liver and pancreas. ${ }^{39}$ BBS is a disease of immotile cilia.

The cilium is anchored in a basal body, a specialised centriole that acts as a microtubule-organising centre. Ciliogenesis and maintenance is orchestrated via the basal body acting in concert with the BBSome, which in turn is modulated by a chaperonin complex and members of the Rab family of proteins. ${ }^{43}$ Together these proteins facilitate intraflagellar transport (IFT). This cellular process of bidirectional movement of particles facilitates the formation and maintenance of the cilium (Figure 2). 


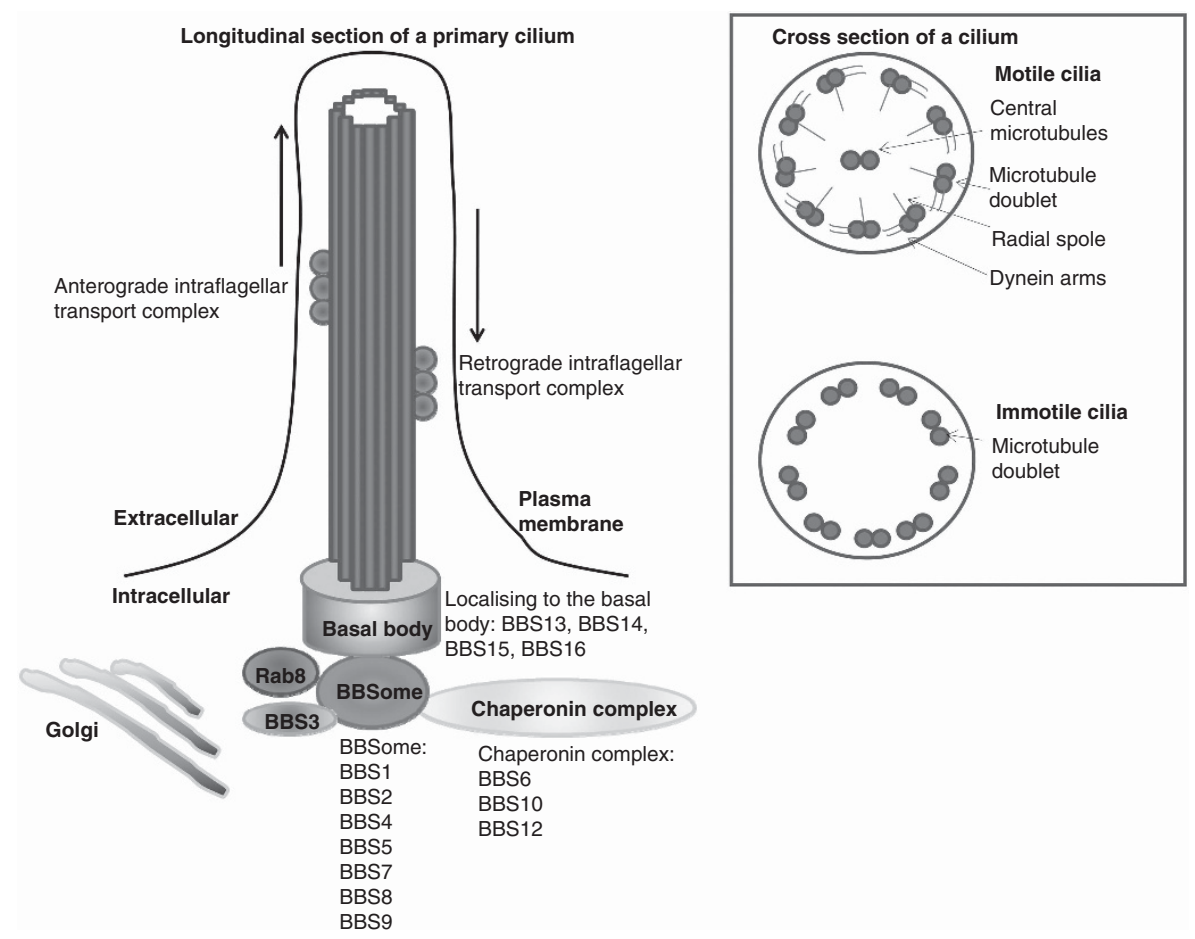

Figure 2 Structure of a cilium. Schematic diagram illustrating a longitudinal section of a primary cilium including the localisation of the proteins encoded by the disease-causing genes in Bardet-Biedl Syndrome. The chaperonin complex mediates assembly of the BBSome, which interacts with BBS3 (a GTPase) and members of the Rab family to organise ciliogenesis and maintenance of the cilium. The cross-section view demonstrates the configuration of motile cilia $(9+2)$ and immotile cilia $(9+0)$.

BBS1, BBS2, BBS4, BBS5, BBS7, BBS8 and BBS9 form the BBSome complex $^{45-47}$ and BBS6, BBS10 and BBS12 form the chaperonin complex. ${ }^{48}$ The remaining known disease-causing genes have variable predicted functions as outlined in Table 2. No phenotypic difference has been ascertained between patients harbouring disease-causing mutations in genes associated with the BBSome compared with individuals with mutations in genes associated with the chaperonin complex.

The molecular pathogenesis of the pleiotropic effects of BBS has been elucidated only in part. The obesity observed in BBS is multifactorial in origin. Energy metabolism appears to be similar in BBS patients and matched obese patients in the general population. ${ }^{49}$ Mouse models demonstrate an association between BBS deficiency, increased food intake and decreased physical activity, and there is evidence of peripheral leptin resistance. ${ }^{50}$ Leptin is a satiety hormone that acts by binding to leptin receptors in the hypothalamus. BBSmutant mice have increased leptin levels. Co-immunoprecipitation experiments show that the BBS1 protein interacts directly with leptin receptors and that the common M390R mutation disrupts this interaction. ${ }^{51,52}$

Marion et $a l^{53}$ found that primary cilia are transiently present in differentiating pre-adipocytes and contain receptors for Wnt and hedgehog pathways. These highly conserved signalling pathways are integral to normal development and act as modulators of adipogenesis. Inhibition of BBS10 and BBS12 induces adipogenesis and suggested that this is secondary to disruption of Wnt signalling, which could not be detected after BBS knock down. ${ }^{53}$

Rod-cone dystrophy is thought to be a consequence of abnormal trafficking across the defective modified cilia connecting the inner and outer segments of photoreceptors leading to apoptosis. ${ }^{50,54,55}$

Receptors for sonic hedgehog signalling are found on cilia in the developing limb buds. ${ }^{55}$ IFT proteins are thought to modulate this pathway ${ }^{39}$ and dysregulation has been associated with the limb malformations observed in ciliopathies. ${ }^{56}$

The mTOR signalling inhibitor rapamycin rescues the renal cysts found in BBS zebrafish morphants, implicating the signalling pathways upstream of mTOR in BBS renal pathology. ${ }^{57}$

Hyposmia/anosmia may be attributed to defective ciliated olfactory epithelium and subfertility in part to defective cilia in sperm cells/oviducts.

BBS was first linked to ciliary dysfunction following the identification of $B B S 8$, which localises to ciliated structures and to basal bodies and centrosomes in cells. ${ }^{58}$ Since then several animal models have confirmed these findings and have furthered current understanding of the function of BBS proteins. Mouse and zebrafish models have identified the role of BBS proteins in Wnt and sonic hedgehog pathways, as well as other signalling pathways. ${ }^{39}$ BBS proteins have been demonstrated to regulate IFT and lipid homoeostasis in worms and modulate intracellular trafficking and centrosomal function in zebrafish. ${ }^{59}$ Mouse models share many of the phenotypic features observed in humans affected with BBS and therefore serve as an excellent mammalian model of the disease. Simons et al ${ }^{60}$ demonstrated this with successful gene therapy preventing photoreceptor death in the BBS4-null murine model. Furthermore, progress in understanding the biological basis for obesity in mouse models may lead to targeted drug therapy for the management of obesity in BBS patients. ${ }^{28,52}$

\section{MANAGEMENT}

A multidisciplinary approach is required to effectively manage this pleiotropic condition. Although research is in progress, there is still no targeted treatment for BBS. Complications associated with BBS should be treated symptomatically as in the general population. Figure $3 \mathrm{a}$ outlines the management of patients with BBS. Figure $3 \mathrm{~b}$ 
outlines the appropriate assessment pathway of patients with suspected BBS who do not fulfil the modified diagnostic criteria for BBS.

Blood pressure should be measured six monthly or more often if there is evidence of hypertension. Antihypertensives and lipid-lowering medication should be prescribed as appropriate. It is recommended that all the patients have at least one baseline renal ultrasound to rule out any obvious malformations. Focused questioning regarding any symptoms of diabetes insipidus is helpful as this often overlooked entity is frequently seen in patients with BBS. Patients with renal impairment should be referred to a nephrologist.

A detailed ophthalmological assessment including an electroretinogram is required to determine the onset and degree of rod-cone dystrophy and to screen for other visual defects such as refractive error, diabetic retinopathy or cataracts. Visual aids and mobility training can significantly improve the quality of life for those who are visually impaired.
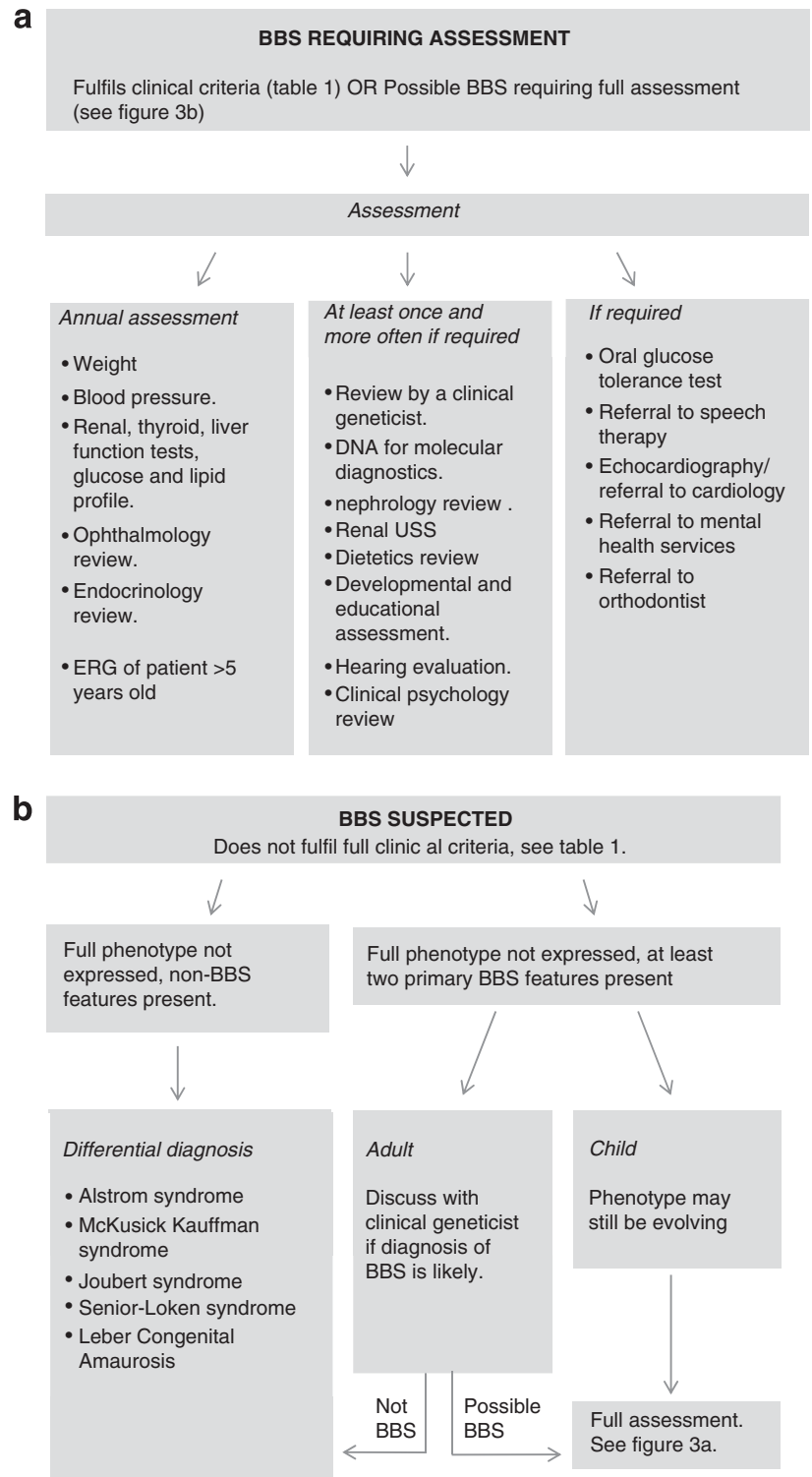

Figure 3 Management of patients with suspected and confirmed BBS (a) Outlines the management of patients with clinically diagnosed BBS. (b) Outlines the appropriate assessment pathway of patients with suspected BBS who do not fulfil the modified clinical criteria for BBS.
Effective weight management is imperative to avoid associated morbidity such as metabolic syndrome to which patients with BBS may be particularly susceptible. Exercise and dietetics review can assist in providing a weight-loss strategy.

Regular developmental and educational assessment is required to ensure that patients gain optimally from their learning environment. It is noteworthy that although early assessment is helpful in placing a child in the best educational environment, it is not always indicative of full adult potential. ${ }^{4}$ Many patients benefit from a review by a clinical psychologist to help with anxiety, depression or behavioural issues, which are more common in BBS.

Endocrinological assessment must include assessment for any signs and symptoms of diabetes mellitus with subsequent oral glucose tolerance testing if appropriate. Assessment of thyroid function, lipid profile and development of secondary sexual characteristics is important. If appropriate, further pituitary function testing can be done and hormone replacement therapy instigated.

Depending on individual need, referral to an orthodontist for assessment of dental crowding/hypodontia or a cardiologist for investigation of any structural cardiac abnormalities may be appropriate.

\section{Genetic counselling}

BBS is generally inherited in an autosomal recessive manner. Although there are multiple cases of triallelic inheritance, these cases are difficult to identify and thought to account for less than $10 \%$ of all cases. ${ }^{5}$ It is therefore appropriate to counsel patients and families according to the traditional autosomal recessive recurrence risk. Hence, any sibling of an affected child has a $25 \%$ risk of being affected, $50 \%$ risk of being an asymptomatic carrier and $25 \%$ of being unaffected and not a carrier.

In families where the disease-causing mutations are known, pre-implantation genetic diagnosis or prenatal testing may be possible. Alternatively, in at-risk families where the mutation is unknown, targeted second trimester sonography may be applied for the visualisation of post-axial polydactyly and renal malformations indicative of a diagnosis of BBS. ${ }^{61,62}$

Patients and parents should be advised of the heterogeneous nature of the condition. The considerable interfamilial and intrafamilial variation hampers predictions about individual educational attainments, visual deterioration or other difficulties associated with BBS. Although infertility is the norm, this cannot be assumed as there are several reports of both men and women with BBS who have had children. ${ }^{4}$

\section{CONCLUSION}

Since the first gene for BBS was identified over a decade ago, there have been extensive developments within the field. A total of 16 disease-causing genes have now been discovered and our understanding of their functional properties has facilitated an insight into the molecular mechanisms underlying ciliary phenotypes in general and BBS in particular. In the coming years, it is likely that other disease-causing genes will be identified and that there will be further improvement of the clinical diagnostic services allowing for faster diagnosis and prenatal testing.

The ability to more accurately predict the level of disability an affected individual is likely to experience may be improved by furthering our understanding of the molecular processes leading to phenotypic variation. Understanding the epigenetic factors that may account for intrafamilial variation and other modifiers of the condition will be imperative in this process.

Elucidation of the molecular pathogenesis of the clinical features of BBS and research into therapeutics may yield novel treatment options that target organ-specific aspects of the condition, such as renal cysts 
or rod-cone degeneration, or have a more general modulating effect on several aspects of the condition.

\section{CONFLICT OF INTEREST}

The authors declare no conflict of interest.

\section{ACKNOWLEDGEMENTS}

We thank Dr Suzanne Rix for her assistance in formatting the photographic images.

1 Laurence JZ, Moon RC: Four cases of 'retinitis pigmentosa' occurring in the same family, and accompanied by general imperfections of development. Obes Res 1995; 3 : 400-403.

2 Bardet G: On congenital obesity syndrome with polydactyly and retinitis pigmentosa (a contribution to the study of clinical forms of hypophyseal obesity). Obes Res 1995; 3: 387-399.

3 Biedl A: A pair of siblings with adiposo-genital dystrophy. Obes Res 1995. 3: 404.

4 Beales PL, Elcioglu N, Woolf AS, Parker D, Flinter FA: New criteria for improved diagnosis of Bardet-Biedl syndrome: results of a population survey. J Med Genet 1999; 36: 437-446

5 Waters AM, Beales PL: Bardet-Biedl Syndrome. GeneReviews [Internet] 1993-2003 (Updated 2011)

6 Riise R, Andreasson S, Borgastrom MK et al: Intrafamilial variation of the phenotype in Bardet-Biedl syndrome. Br J Ophthalmol 1997; 81: 378-385.

7 Katsanis N, Ansley SJ, Badano JL et al: Triallelic inheritance in Bardet-Biedl syndrome, a Mendelian recessive disorder. Science 2001; 293: 2256-2259.

8 Farag TI, Teebi AS: High incidence of Bardet Biedl syndrome among the Bedouin. Clin Genet 1989; 36: 463-464.

9 Green JS, Parfrey PS, Harnett JD et al: The cardinal manifestations of Bardet-Biedl syndrome, a form of Laurence-Moon-Biedl syndrome. N Engl J Med 1989; 321: 1002-1009.

10 Webb MP, Dicks EL, Green JS et al: Autosomal recessive Bardet-Biedl syndrome: firstdegree relatives have no predisposition to metabolic and renal disorders. Kidney Int 2009; 76: 215-223.

11 Hamel CP: Cone rod dystrophies. Orphanet J Rare Dis 2007; 2: 7.

12 Baker K, Beales PL: Making sense of cilia in disease: the human ciliopathies. Am J Med Genet C Semin Med Genet 2009; 151C: 281-295.

13 Adams NA, Awadein A, Toma HS: The retinal ciliopathies. Ophthalmic Genet 2007; 28: $113-125$.

14 Tobin JL, Beales PL: Bardet-Biedl syndrome: beyond the cilium. Pediatr Nephrol 2007; 22: 926-936.

15 Hjortshoj TD, Gronskov K, Philp AR et al: Bardet-Biedl syndrome in Denmark - report of 13 novel sequence variations in six genes. Hum Mutat 2010; 31: 429-436.

16 Rooryck C, Lacombe D: Bardet-Biedl syndrome. Ann Endocrinol (Paris) 2008; 69: 463-471.

17 Moore SJ, Green JS, Fan Y et al: Clinical and genetic epidemiology of Bardet-Biedl syndrome in Newfoundland: a 22-year prospective, population-based, cohort study. Am J Med Genet A 2005; 132: 352-360.

18 Putoux A, Attie-Bitach T, Martinovic J, Gubler MC: Phenotypic variability of BardetBiedl syndrome: focusing on the kidney. Pediatr Nephrol 2012; 27: 7-15.

19 Deveault C, Billingsley G, Duncan JL et al: BBS genotype-phenotype assessment of a multiethnic patient cohort calls for a revision of the disease definition. Hum Mutat 2011; 32: 610-619.

20 O'Dea D, Parfrey PS, Harnett JD, Hefferton D, Cramer BC, Green J: The importance of renal impairment in the natural history of Bardet-Biedl syndrome. Am J Kidney Dis 1996; 27: 776-783.

21 Marion V, Schlicht D, Mockel A et al: Bardet-Biedl syndrome highlights the major role of the primary cilium in efficient water reabsorption. Kidney Int 2011; 79: 1013-1025.

22 Beales PL, Warner AM, Hitman GA, Thakker R, Flinter FA: Bardet-Biedl syndrome: a molecular and phenotypic study of 18 families. J Med Genet 1997; 34: 92-98.

23 Elbedour K, Zucker N, Zalzstein E, Barki Y, Carmi R: Cardiac abnormalities in the Bardet-Biedl syndrome: echocardiographic studies of 22 patients. Am J Med Genet 1994; 52: 164-169.

24 de Pontual L, Zaghloul NA, Thomas S et al: Epistasis between RET and BBS mutations modulates enteric innervation and causes syndromic Hirschsprung disease. Proc Nat Acad Sci USA 2009; 106: 13921-13926.

25 Kulaga HM, Leitch CC, Eichers ER et al: Loss of BBS proteins causes anosmia in humans and defects in olfactory cilia structure and function in the mouse. Nat Genet 2004; 36: 994-998.

26 Slavotinek AM, Biesecker LG: Phenotypic overlap of McKusick-Kaufman syndrome with Bardet-Biedl syndrome: a literature review. Am J Med Genet 2000; 95: 208-215.

27 Croft JB, Morrell D, Chase CL, Swift M: Obesity in heterozygous carriers of the gene for the Bardet-Biedl syndrome. Am J Med Genet 1995; 55: 12-15.

28 Guo DF, Rahmouni K: Molecular basis of the obesity associated with Bardet-Biedl syndrome. Trends Endocrinol Metab 2011; 22: 286-293.

29 Beales PL, Reid HA, Griffiths MH, Maher ER, Flinter FA, Woolf AS: Renal cancer and malformations in relatives of patients with Bardet-Biedl syndrome. Nephrol Dial Transplant 2000; 15: 1977-1985.
30 Kim LS, Fishman GA, Seiple WH, Szlyk JP, Stone EM: Retinal dysfunction in carriers of Bardet-Biedl syndrome. Ophthalmic Genet 2007; 28: 163-168.

31 Benzinou $\mathrm{M}$, Walley $\mathrm{A}$, Lobbens $\mathrm{S}$ et al: Bardet-Biedl syndrome gene variants are associated with both childhood and adult common obesity in French Caucasians. Diabetes 2006; 55: 2876-2882.

32 Billingsley G, Deveault C, Heon E: BBS mutational analysis: a strategic approach. Ophthalmic Genet 2011; 32: 181-187.

33 Eichers ER, Lewis RA, Katsanis N, Lupski JR: Triallelic inheritance: a bridge between Mendelian and multifactorial traits. Ann Med 2004; 36: 262-272.

34 Beales PL, Badano JL, Ross AJ et al: Genetic interaction of BBS1 mutations with alleles at other BBS loci can result in non-Mendelian Bardet-Biedl syndrome. Am J Hum Genet 2003; 72: 1187-1199.

35 Badano JL, Kim JC, Hoskins BE et al: Heterozygous mutations in BBS1, BBS2 and BBS6 have a potential epistatic effect on Bardet-Biedl patients with two mutations at a second BBS locus. Hum Mol Genet 2003; 12: 1651-1659.

36 Katsanis N, Eichers ER, Ansley SJ et al: BBS4 is a minor contributor to Bardet-Bied syndrome and may also participate in triallelic inheritance. Am J Hum Genet 2002; 71: 22-29.

37 Zaghloul NA, Katsanis N: Mechanistic insights into Bardet-Biedl syndrome, a model ciliopathy. J Clin Invest 2009; 119: 428-437.

38 Karmous-Benailly $\mathrm{H}$, Martinovic J, Gubler MC et al: Antenatal presentation of Bardet-Biedl syndrome may mimic Meckel syndrome. Am J Hum Genet 2005; 76: 493-504.

39 Gerdes JM, Davis EE, Katsanis N: The vertebrate primary cilium in development, homeostasis, and disease. Cell 2009; 137: 32-45.

40 Leitch CC, Zaghloul NA, Davis EE et al: Hypomorphic mutations in syndromic encephalocele genes are associated with Bardet-Biedl syndrome. Nat Genet 2008; 40: 443-448.

41 Riise R, Tornqvist K, Wright AF, Mykytyn K, Sheffield VC: The phenotype in Norwegian patients with Bardet-Biedl syndrome with mutations in the BBS4 gene. Arch Ophthalmol 2002; 120: 1364-1367.

42 Heon E, Westall C, Carmi R et al: Ocular phenotypes of three genetic variants of Bardet-Biedl syndrome. Am J Med Genet A 2005; 132A: 283-287.

43 Waters AM, Beales PL: Ciliopathies: an expanding disease spectrum. Pediatr Nephrol 2011; 26: 1039-1056.

44 Waters AM, Beales PL: Ciliopathies: an expanding disease spectrum. Pediatr Nephrol 2011; 26: 1039-1056.

45 Loktev AV, Zhang Q, Beck JS et al: A BBSome subunit links ciliogenesis, microtubule stability, and acetylation. Dev Cell 2008; 15: 854-865.

46 Nachury MV, Loktev AV, Zhang $Q$ et al: A core complex of BBS proteins cooperates with the GTPase Rab8 to promote ciliary membrane biogenesis. Cell 2007; 129: 1201-1213.

47 Jin H, Nachury MV: The BBSome. Curr Biol 2009; 19: R472-R473.

48 Seo S, Baye LM, Schulz NP et al: BBS6, BBS10, and BBS12 form a complex with CCT/TRiC family chaperonins and mediate BBSome assembly. Proc Natl Acad Sci USA 2010; 107: 1488-1493

49 Grace C, Beales P, Summerbell C et al: Energy metabolism in Bardet-Biedl syndrome. Int J Obes Relat Metab Disord 2003; 27: 1319-1324.

50 Sheffield VC: The blind leading the obese: the molecular pathophysiology of a human obesity syndrome. Trans Am Clin Climatol Assoc 2010; 121: 172-181.

51 Rahmouni K, Fath MA, Seo S et al: Leptin resistance contributes to obesity and hypertension in mouse models of Bardet-Biedl syndrome. J Clin Invest 2008; 118 : 1458-1467.

52 Seo S, Guo DF, Bugge K, Morgan DA, Rahmouni K, Sheffield VC: Requirement of Bardet-Biedl syndrome proteins for leptin receptor signaling. Hum Mol Genet 2009; 18: 1323-1331.

53 Marion V, Stoetzel C, Schlicht D et al: Transient ciliogenesis involving Bardet-Biedl syndrome proteins is a fundamental characteristic of adipogenic differentiation. Proc Natl Acad Sci USA 2009; 106: 1820-1825.

54 Nishimura DY, Fath M, Mullins RF et al: Bbs2-null mice have neurosensory deficits, a defect in social dominance, and retinopathy associated with mislocalization of rhodopsin. Proc Natl Acad Sci USA 2004; 101: 16588-16593.

55 Mockel A, Perdomo Y, Stutzmann F, Letsch J, Marion V, Dollfus H: Retinal dystrophy in Bardet-Biedl syndrome and related syndromic ciliopathies. Prog Retin Eye Res 2011; 30: 258-274

56 Bimonte $S$, De Angelis A, Quagliata $L$ et al: Ofd 1 is required in limb bud patterning and endochondral bone development. Dev Biol 2011; 349: 179-191.

57 Tobin JL, Beales PL: Restoration of renal function in zebrafish models of ciliopathies. Pediatr Nephrol 2008; 23: 2095-2099.

58 Ansley SJ, Badano JL, Blacque OE et al: Basal body dysfunction is a likely cause of pleiotropic Bardet-Biedl syndrome. Nature 2003; 425: 628-633.

59 Blacque OE, Leroux MR: Bardet-Biedl syndrome: an emerging pathomechanism of intracellular transport. Cell Mol Life Sci 2006; 63: 2145-2161.

60 Simons DL, Boye SL, Hauswirth WW, Wu SM: Gene therapy prevents photoreceptor death and preserves retinal function in a Bardet-Biedl syndrome mouse model. Proc Natl Acad Sci USA 2011; 108: 6276-6281.

61 Dar P, Sachs GS, Carter SM, Ferreira JC, Nitowsky HM, Gross SJ: Prenatal diagnosis of Bardet-Biedl syndrome by targeted second-trimester sonography. Ultrasound Obstet Gynecol 2001; 17: 354-356.

62 Cassart M, Eurin D, Didier F, Guibaud L, Avni EF: Antenatal renal sonographic anomalies and postnatal follow-up of renal involvement in Bardet-Biedl syndrome. Ultrasound Obstet Gynecol 2004; 24: 51-54. 